Southern Methodist University

SMU Scholar

2006

\title{
Questioning Our Principles: Anthropological Contributions to Ethical Dilemmas in Clinical Practice
}

\author{
Carolyn Sargent \\ Washington University in St Louis \\ Carolyn Smith-Morris \\ smithmor@mail.smu.edu
}

Follow this and additional works at: https://scholar.smu.edu/hum_sci_anthropology_research

Part of the Bioethics and Medical Ethics Commons, Ethics and Political Philosophy Commons, and the Social and Cultural Anthropology Commons

\section{Recommended Citation}

Sargent, Carolyn and Smith-Morris, Carolyn, "Questioning Our Principles: Anthropological Contributions to Ethical Dilemmas in Clinical Practice" (2006). Anthropology Research. 2.

https://scholar.smu.edu/hum_sci_anthropology_research/2

This document is brought to you for free and open access by the Anthropology at SMU Scholar. It has been accepted for inclusion in Anthropology Research by an authorized administrator of SMU Scholar. For more information, please visit http://digitalrepository.smu.edu. 
Southern Methodist University

SMU Digital Repository

Anthropology Research

$1-1-2006$

\section{Questioning Our Principles: Anthropological Contributions to Ethical Dilemmas in Clinical Practice}

Carolyn Sargent

Carolyn Smith-Morris 
Special Section: The Power of Choice: Autonomy, Informed Consent, and the Right to Refuse

\title{
Questioning Our Principles: Anthropological Contributions to Ethical Dilemmas in Clinical Practice
}

\author{
CAROLYN SARGENT and CAROLYN SMITH-MORRIS
}

This paper presents an analysis of the applicability of a principalist approach for a global, or cross-cultural, bioethics. We focus especially on the principle of individual autonomy, a core value in ethical discourse. We echo some longstanding criticisms of other anthropologists, sociologists, and many medical ethicists that the individualistic approach to autonomy is a Euro-American value and cannot be ethically applied in all settings. As a remedy, we suggest an adaptation of Kleinman's Explanatory Model approach to questions of decisionmaking. ${ }^{1}$ We argue that the analysis and resolution of ethical dilemmas might also benefit from forms of pedagogy that integrate anthropological and other social science perspectives, and the incorporation of ethnographic techniques in ethical practice.

We begin our discussion with a case.

\section{Case 1}

Mr. $\mathrm{R}$ is an Asian man with seriously compromised lung function, hospitalized for several months, currently on a ventilator. The ICU medical team wants to remove him from life support, but the family, consisting of his wife and two daughters, have so far declined.

An ethics consult is arranged to mediate between the ICU personnel and the family. The family has been in the United States for about six years and the wife does not speak English. An interpreter is present. A physician begins by asking the wife, through the interpreter, "Do you think your husband would want to be like this? Do you think he is happy?" He then shows an X ray of the patient's lungs to demonstrate the gravity of his condition.

A bilingual friend arrives. The physicians present options: Place the patient on a ward with a DNR order and the understanding that he will not be moved back to the ICU or send him home with a ventilator (although the family has already said that everyone works extensive hours and no one will be available to stay with him). Because he is not a citizen, there are no nursing homes available (in this state) for transferring a ventilator-dependent patient. The family friend translates for his wife: "She is his wife, she cannot say whether he is happy. As his wife, she does not know what he would want. It is not possible for her to decide. Also, she believes that as a Catholic, she cannot choose to

We appreciate the extensive efforts of Michelle Amoruso, doctoral candidate in anthropology, who assisted with the preparation of the manuscript. 


\section{Carolyn Sargent and Carolyn Smith-Morris}

withdraw him from life support. She wants him to stay in the ICU on the machine."

At this point in the consult, the medical team is visibly angry, stating in English that his condition is irreversible and they need his room for someone who might live. It's an economic issue. They also comment that the family is wrong in their understanding of church policy on withdrawing life support.

What can ethnographic or cultural analysis add to our understanding of this or other such cases? There are a variety of issues in this case that easily lend themselves to anthropological exposition. Mutual exploration of the following themes by ethicists and anthropologists might generate rich material to shape the resolution of this dilemma:

- Patriarchal family and customary gender roles

- Individual versus family (collective) focus of decisions

- Negotiation of religious interpretations

- Discussion of death-how appropriate is open discussion?

- Truth-telling as acceptable or not

- Issues of distributive justice (how do and how should we respond to the reality that, if this patient lived in New York or Illinois, he could, as a noncitizen, be moved to a nursing home on life support, as his family wishes?).

Our fundamental objective in this discussion is to interrogate the problematic biomedical/Euro-American bias toward the individual and overreliance on a Euro-American bioethics approach.

\section{Introduction to the Four Principles Approach}

The Four Principles approach in bioethics, made famous by Beauchamp and Childress in the five editions of their classic text on biomedical ethics, is the hallmark of the discipline, the theoretical center from which much of the field of bioethics has grown. ${ }^{2}$ Although much critiqued by ethicists from diverse standpoints and by anthropologists (e.g., physician-anthropologists Kleinman and Helman argue cogently against assuming the relevance of Eurocentric premises in clinical practice), these principles continue as implicit, if not explicit, premises underlying analysis of ethical issues and decisionmaking in clinical settings. ${ }^{3}$ Indeed, a cursory review of recent scholarship immediately illustrates the enduring reliance on Beauchamp and Childress for an ethical framework for theory and practice. ${ }^{4} \mathrm{O}^{\prime}$ Neill, for example, opens a discussion on "Practical Principles, Practical Judgment" ${ }^{5}$ by stating that the most wellknown approach to bioethical reasoning that appeals to principles remains that of Beauchamp and Childress. ${ }^{6}$ Similarly, Annas, writing in the New England Journal of Medicine, critiques the recent Terri Schiavo case, and emphasizes the importance of patients' rights, as consistent with American values and constitutional traditions. ${ }^{7}$ He quotes the Massachusetts Supreme Judicial Court on the sanctity of individual choice and self-determination as fundamental constituents of life. ${ }^{8}$ The principle of individual autonomy as core to ethical considerations is again reiterated.

As an ethical framework, the principles approach is an "attempt[s] to identify and justify" a given set of moral norms for the "guidance of and evaluation 


\section{Questioning Our Principles}

of conduct." 9 The principles are described as "four clusters of moral principles" and include the following:

1. Respect for autonomy-a norm of respecting the decisionmaking capacities of individual persons

2. Nonmaleficence-a norm of avoiding the causation of harm

3. Beneficence-a group of norms for not only "doing good" but balancing those good works against the risks and costs associated with the effort

4. Justice-a group of norms for distributing benefits, risk, and costs fairly. ${ }^{10}$

Beauchamp and Childress have settled on these principles using assumptions about a "common morality" which they define as "[T]he set of norms that all morally serious persons share ... [It] contains moral norms that bind all persons in all places; no norms are more basic to moral life"11 and it is "the morality that serves as our common heritage." 12

To utilize these principles in practice, Beauchamp and Childress outline several rules. They insist that, "Principles are general norms that leave considerable room for judgment in many cases." More detailed "rules" and "judgments" are therefore required and "function as precise action guides that inform us in each circumstance how to act." ${ }^{13}$ Some of these rules might include the following:

- Substantive Rules (truth-telling, confidentiality, privacy, physician-assisted suicide, informed consent, and the like)

- Authority Rules (who may and should perform actions)

- Procedural Rules (rules that establish procedures to be followed, e.g., for determining eligibility for scarce resources, for reporting grievances).

Finally, Beauchamp and Childress introduce elements to contend with nonuniversal norms and human idiosyncrasies-these include rights, the idea of moral character, virtues, the greatest balances of right and wrong, competing obligations, and even emotions. All of these are considered vital to the theory and to practical applications, although they are handmaidens to the Four Principles.

In our discussion, we will focus on the culturally Euro-American reification of the Individual, which is so fundamental to the four principles as to limit its utility for communication in ethical conflicts, even though the U.S. legal system rests on the concept of individual rights. The principle of justice affirms the isomorphic relationship between a principalist approach and Euro-American jurisprudence, yet for patients and families of any cultural or class background, the critical meanings associated with an ethical dilemma may be situated outside the parameters of this narrow ethical/legal domain.

What we will propose is simultaneous attention to the individual and cultural factors in questions of autonomy, patient rights, and the decisionmaking process.

Following Marshall and Koenig, we do not "dispute the relevance of abstract principles in bioethics ... [but direct our] concern as anthropologists [to] the everyday practices derived from these principles, practices that often fall short of realizing the normative intent of the principles themselves." 14 In particular, we (and they) are troubled by the unexamined "promulgation of a Westernized bioethics that fosters only an illusion of global consensus about the morality of medical practice." 15 


\section{Carolyn Sargent and Carolyn Smith-Morris}

As anthropologists, we suggest that the subject of medical ethics should be the cultural construction of morality, particularly morality surrounding health and disease. Empiricism is requisite for a just and informed bioethics-a descriptive bioethics that grounds each case in its cultural, historical, and political-economic contexts. There are two main critiques we make: that the individual is so prioritized and central as to become an unquestioned presumption of care and that the notion of a common morality is a false, if not a culturally imperialist, idea that justifies the devaluation of locally meaningful moralities. Were one to explore it at a sufficiently high level of abstraction, one might be able to identify areas of widely shared values. However, when one gets to the specifics of when certain moral values can be imposed, then what seem in the abstract to be morally universals quickly become culturally specific. For example, when is it acceptable to kill? Is it ever acceptable to rape? What constitutes child abuse? ${ }^{16}$

\section{Reification of the Individual}

The reification of individual choice in the principalist approach is evident in several places:

- in notions of the autonomy of any given individual

- in the complete preoccupation with a focal "patient," the single suffering individual

- and even in biomedically defined "cases" (which have a hegemonically determined beginning and end).

These assumptions, long the target of critique by medical anthropologists as well as by some medical ethicists, reinforce specific cultural notions about the target of care, the definition of the patient, and the responsibilities of various parties involved. These assumptions are increasingly inappropriate in healthcare, where patients frequently come from cultural backgrounds different from their doctors. Helman, a physician and anthropologist, notes that biomedicine focuses on the individual patient, or even the individual organ, while ignoring wider familial, social, and economic issues that render consensus or problem resolution difficult to achieve. ${ }^{17}$

Anthropologist Patricia Marshall has suggested that, "Bioethics practices that celebrate only autonomy, with its emphasis on choice, and downplay social and economic constraints on individual agency, are out of touch with health-care realities in the U.S., as well as globally. The changed discourse-doctor becomes provider and patient becomes consumer-reflects fundamental, systemic problems characteristic of market-driven medicine." 18 Marshall and Koenig argue that conventional individual-focused bioethics practices by themselves, and without attention to the broader context in which individual decisions are made, may be inadequate.

Beauchamp and Childress respond to attacks leveled against the Autonomy Principle by insisting on the individual right to determine the form and content of care. They write:

We defend a principle of respect for autonomy with a correlative right to choose (not a mandatory duty to choose). (A study they describe of 


\section{Questioning Our Principles}

800 subjects from 4 different ethnic groups-Korean American, Mexican American, European American, and African American-showed ethnicity to be a primary correlate with attitudes toward disclosure and decision-making. But, they go on) ... [e]ven if the patient delegates [their decisionmaking] right to someone else, the choice to delegate is itself autonomous. ${ }^{19}$

Patients whose cultural or family context renders them unable or unwilling to make decisions about their own care can, according to the principle of autonomy, choose to refuse information about their health and direct that others will make decisions for them. How and by whom these interactions will occur is left to guesswork. As Beauchamp and Childress respond, the only obligation is on the physician.

There is a fundamental obligation to ensure that patients have the right to choose, as well as the right to accept or to decline information.... The tricky practical question is whether it is possible to inform patients of their rights to know and to decide without compromising their systems of belief and value or otherwise disrespecting them. 20

A "tricky practical question" indeed. Patient decisionmaking has become an obligation in the lived world of hospital and managed care and we suggest that a truly moral stance cannot ignore the context within which ethical theories are played out and must acknowledge the reality in which its ideals must be rooted.

That is, to propose as a theoretical principle a concept (i.e., non-obligatory autonomy) that is almost universally disregarded must call into question the validity of the theory. If patient autonomy is viewed in the vast majority of settings as not just a right but an obligation of patients, then this problem demands both theoretical and pragmatic remedies. The Four Principles approach offers neither.

As we have noted, Beauchamp and Childress do claim sensitivity to the social determinants of morality and to the function of judgment applied in individual cases. They say that the four principles are general norms that leave "considerable room for judgment in many cases." In addition, they acknowledge that moral virtues, paramount in driving decisions for each clinical case, are socially determined. Yet, they say, we do not approach those cases tabula rasa but with norms and assumptions informed by culture, history, and the like.

We suggest that proponents of the principles framework approach their cases and theory with the same culturally informed assumptions. They have built a theory that so reifies the individual that no room is left available in cases for judgments that might contradict these fundamental assumptions.

\section{Common Morality}

The idea of a common, universal morality is not something readily accepted by anthropologists, and it has come under considerable attack by bioethicists as well. Beauchamp and Childress acknowledge this when they write:

the common morality's norms do require interpretation if we are to have workable practical ethics. Such interpretation is often subject to vigorous dispute in order to resolve particular problems. ${ }^{21}$ 


\section{Carolyn Sargent and Carolyn Smith-Morris}

They acknowledge that not all people accept these norms, but that all serious people accept them, presumably serious Euro-Americans.

Recent conversations have attempted to align the "common morality" with notions of basic human rights. Thus, there are basic human rights (roughly equivalent to the notion of a common morality) and there is communityspecific morality, which takes into account local variation in what is considered appropriate moral behavior and motivation. This distinction is far too general to guide clinical practice.

So, to employ the Four Principles approach, we must accept that humans share innate (i.e., universal) processes or characteristics that eventuate in a common morality. Anthropologists, trained to be sensitive to the diversity of human thoughts and values, typically eschew such assumptions about innate or universal. Virtually all schools of anthropology entail an acceptance of at least a weak form of descriptive relativism. Normative relativism, favored by some anthropologists, goes a step further in asserting that, because cultures judge each other according to their own internal standards, there are no universal standards to judge between cultures. ${ }^{22}$

Some of the assumptions made under the banner of common morality include: individualistic thinking - rather than communal, family, or some other form of thought or orientation; the linearity of time-rather than cyclical time; the universality of truth-telling; informed consent; definitions of personhood; ownership and transplantation of body parts; and withholding or withdrawing life-sustaining treatments as moral virtues. ${ }^{23}$

Ethnographies (i.e., anthropological descriptions and analyses of specific cultures) abound that challenge the appropriateness of these "beliefs." These assumptions become very apparent and, often, problematic in end-of-life care. Barbara Koenig, for example, says that advance directive assume an ideal patient with

- a clear understanding of the illness, prognosis, and treatment options shared with the medical team

- a temporal orientation to the future and a desire to maintain control over the future

- a perception of freedom of choice

- a willingness to discuss death openly. ${ }^{24}$

This largely represents educated, middle/upper-middle class Americans. But Smith-Morris's research among elderly, predominantly Anglo adults in Arizona suggests that even these privileged citizens are ill prepared for a more likely end-of-life scenario. ${ }^{25}$

\section{Alternatives and What Anthropology/ists Can Offer}

What principles or ethical theory could better address this plurality? A variety of alternative theories and approaches exist now for bioethicists to choose from, for example, casuistry, relationship-based approaches, utilitarianism, character or virtue ethics, and communitarianism. But few of these provide for the type and depth of descriptive context we propose, and none address authoritative knowledge and the power structures within which decisions about health and healthcare are made. So if none of these options do it, what 


\section{Questioning Our Principles}

exactly can anthropologists add? Why would ethnographic data lead to more ethical practice?

Case 2

To explore anthropologists' potential roles in improving bioethics, we offer a brief second case.

A young adult male is brought to the ER having swallowed a Pentium 4 computer chip. He has a history of psychotic episodes. He now has bowel obstruction but refuses surgery, apparently wanting to keep the mechanism. A psychiatry consult determines that he is not competent to make decisions and he is sent to surgery.

Once there, an OR authority questions him to determine if he is "oriented $\times 3$." He knows his name, the day, and the president. The OR nurse then overrides the surgery decision, having determined to her own satisfaction that he is competent.

In an ethics consult to discuss the decisionmaking process in this case, the psychiatry residents mention that although the patient is mildly retarded, this was not a factor in their determination that he lacked the capacity to make a decision about surgery. The OR nurse then vigorously states that if she had known he was retarded, she would have never questioned the psychiatry decision.

So this case raises questions concerning cultural constructs of mental illness and mental functioning that affect both popular thinking and understandings of biomedical professionals outside psychiatry. It appears from the discussion that the concept of "retardation" is less contested than that of psychosis, or delusional states, even within biomedicine.

An ethnography of nurses and doctors in different specialties and their understandings of psychosis might provide us with information that could then remedy some of these issues, thus leading to workshops on what it means to be delusional, especially in relation to functioning effectively in some domains of social life. This, in tum, could enhance the understanding of how decisionmaking competence is determined and the limits of individual autonomy. That is, anthropologists can add ethnographic detail, informing ethicists and clinicians of the personal narratives, cultural meanings, and local moralities that shape decisionmaking. We should note that the concept of "personal narrative" might suggest an idiosyncratic, subjective account. However, as Kaufman cogently argues, narratives are constructed from shared understandings of the cultural world, and as such, they identify important cultural and structural features that shape individual moral thought and feeling. ${ }^{26}$

\section{Authoritative Knowledge}

A theory of biomedical ethics must step beyond the bounds of hegemonic assumptions. The principalist approach re-creates and reinforces the primacy of individualism, rationality, and a bounded temporal experience of disease while failing to acknowledge power relations implicit in biomedical decisionmaking. Authoritative knowledge is produced, displayed, resisted, and challenged in social, clinical, and political interactions. Much research, including Sargent's, demonstrates the links between control of technology and the hierarchy of 
relations between specialists and patients. ${ }^{27}$ But research also shows the possibility for interactional cooperation (alliances) and accommodation in the clinical setting. The constitution of authoritative knowledge is an ongoing social process; it constructs and reflects power relationships within a community of practice. The process by which this occurs is such that all participants tend to see the current social order as "the way things obviously are."

These hegemonic forces are what Beauchamp and Childress seem most blind to. Questions of power in moral and ethical dilemmas-power between practitioners and patients, power between different types or fields of practitioners, power within families and cultures-are marginalized and left unchallenged.

Many bioethicists and clinicians might argue that they have moved beyond a rigid application of principles to resolve ethical dilemmas, and rather, engage in flexible negotiations, indeed in a form of therapeutic intervention in the resolution of such conflicts. We need to ask, however, what is the ultimate objective of these negotiations? Often, the implicit, if not explicit, goal is to convey to the patient and/or family the preferred decision of the clinician, based presumably on medical expertise or institutional concerns about liability.

Should we consider these "flexible negotiations" a form of benevolent paternalism? We suggest the importance of acknowledging the authoritative knowledge of the physician and other biomedical practitioners. Physicians, whose moral weight and perceived scientific expertise are linked to formidable medical technologies, are in a position of power, and rarely in an egalitarian, collaborative interaction. ${ }^{28}$ How authoritative knowledge is produced and displayed in ethical consultations is a question that anthropologists could usefully address.

A grounded approach to bioethics can be generated by ethnography. Understanding the patient's explanatory model can help bioethicists as well as biomedical practitioners explain that system to patients/families so that they can make better informed decisions that the system, in fact, requires them to make. For example, the value placed on the individual in the United States creates not an accidental correspondence between the ethics approach and the legal protocol; the predominant ethics approach is isomorphic with the legal system (J. Sadler, personal communication).

Do ethics consults actually do broader cultural work of furthering a shared moral order in the context of a multicultural society? If we inform only the system of the patient's model, we are reinforcing medicine as a privileged domain of moral discourse. However, if we inform patients of cultural details, power dynamics, and institutional requirements of biomedicine, then they can ask more nuanced questions and have a better chance of truly participating in an asymmetric therapeutic interaction.

We propose an alternative for moving between a larger cultural, historical, or family structure and context to the notion of an individual as the unit of analysis. We would first redefine "the case."

The boundedness of the case is another unexamined assumption fundamental in the principalist approach and closely related to, if not produced by, the reification of individuals. Helman, for instance, observes that biomedicine is unique in its imposition on the patient of a linear narrative structure, the "case history." 29

The clinical case-the identified patient, other relevant decisionmakers, even the parameters in a temporal sense (when illness began and when it ends) -is 


\title{
Questioning Our Principles
}

a manufactured product; a certain telling of the story among many other possible tellings. ${ }^{30}$ Relegated to the background are the economic and social contexts of the patient's life, cultural factors in the recognition and meaning of illness, potential stigma, simultaneous (other) healing efforts, and so forth. Even casuist and hermeneutic approaches, known for their strident rejection of principalism, fail to expand the parameters of the case itself.

A procedurally simple change, implemented not after or in the midst of a crisis (as we so often see in ethics consults), but at the initial presentation for carethat is, as part of the intake process-would allow the alignment of patient and clinician expectations to occur at a more natural point in the relationship. This approach would involve eliciting the patient and family's explanatory model. An explanatory model, as first proposed by Arthur Kleinman and later elaborated by him with Leon Eisenberg and Byron Good, are the notions about an episode of sickness and its treatment that are employed by all those engaged in the clinical process. Barry Hoffmaster adds that patient models reflect social class, cultural beliefs, education, occupation, religious affiliation, and past experience with illness and healthcare. ${ }^{31}$ Returning to the issue of power differentials, Helman argues that the power invested in clinicians as a result of their training and technical expertise often allows them to shape the patient's explanatory model to fit the medical model, rather than allowing the patient's perspective on illness to emerge. ${ }^{32}$ Correspondingly, Kleinman, in his groundbreaking book on medicine as a cultural system, states that

\begin{abstract}
the most difficult aspect of clinical practice to teach to medical students, interns, and residents is how to elicit and evaluate objectively patient beliefs and values with respect to their illnesses and treatments and to negotiate with (or translate between) these differing perspectives, in the same way an advisor gives expert advice to an advisee, who retains the right to accept, alter, or reject that advice. ${ }^{33}$
\end{abstract}

Collecting the explanatory model can be done through a series of questions like those in Figure 1.

Adapting this model for a more ethically neutral approach to healthcare decisionmaking might look like the model shown in Figure 2 . That is, it would begin with aspects of decisionmaking and the values and beliefs guiding care.

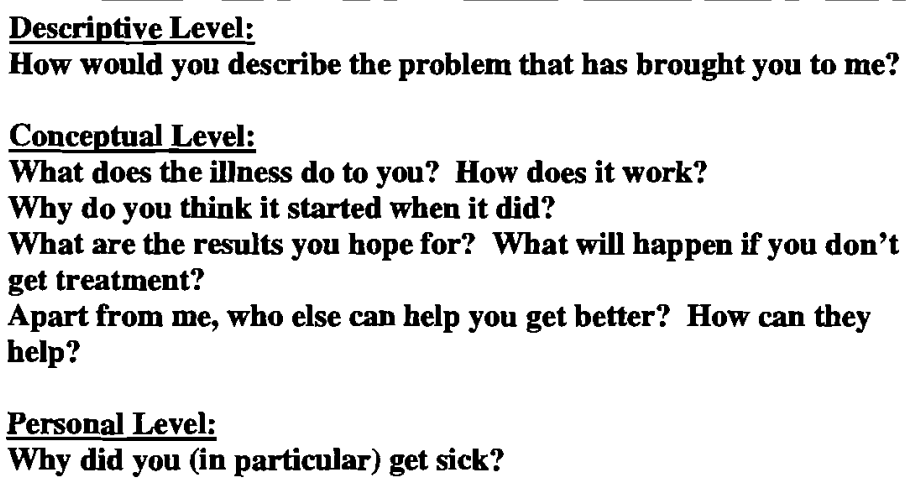

Figure 1. An explanatory model. 
Carolyn Sargent and Carolyn Smith-Morris

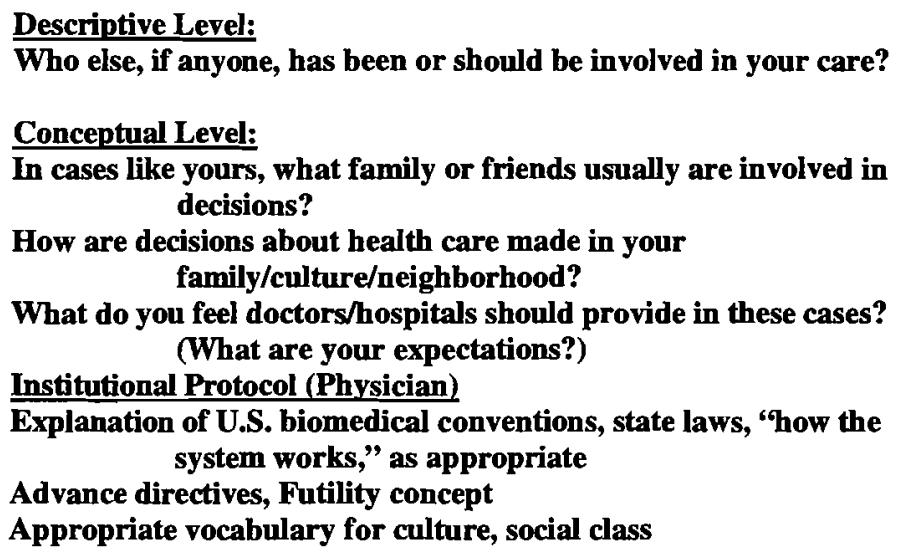

Figure 2. An ethically neutral model.

How might ethical decisions change if cases came to be viewed as life stories, family events, or other ongoing narrative? A grounded approach that is informed by ethnographic information would reliably attend to the structural, institutional, and procedural barriers that worsen - and in many cases produceethical dilemmas in care. Armed with this information, clinicians and institutions would at least have insight into problematic arenas requiring changes.

A final case will demonstrate what we suggest.

\section{Case 3}

A doctor writes to an ethics committee requesting a discussion of patients who behave abusively to doctors and nurses. The committee, composed of doctors, nurses, social workers, chaplains, and community representatives, agrees that this is an important issue. The anthropologist present asks what is this abuse (verbal and sometimes physical) and who are the abusers? The response from one prominent and respected physician is "generic scumbags." The anthropologist suggests a survey in which when a case arises, the following information is reported to a central source: age, sex, ethnicity of patient, medical condition, and circumstances/context when incident occurred. Committee members are not interested, deciding rather to ask for a consult with psychiatry on how to manage disruptive patients. For them, a key ethical issue is whether it is acceptable to call security for such a patient (with exceptions made for someone with dementia or on drugs).

Management of the abusive patients, certainly a reasonable concern, becomes the core issue of discussion. What we lack, however, are any data on precisely who these "abusers" might be (we might speculate on age, sex, insurance status) and in what sorts of situations abusive behavior might emerge. Eliciting and analyzing this information might then provide us with a means to address the fundamental, underlying causes of disruptive behavior, and therefore to identify possible structural factors implicated in these scenarios. 


\section{Questioning Our Principles}

\section{Closing}

Bioethics is a field now dominated by premises of Western philosophical thought: principles and rights-based approaches that have reinforced a "pervasive reductionism, utilitarianism, and ethnocentrism in the field.." ${ }^{34}$ The original bioethics was intended to empower patients in the context of a rights-based approach, which would allow patients to reclaim power from biomedical expertise. It is ironic that this effort to generate empowerment has had such mixed results. ${ }^{35}$

We suggest that incorporating an ethnographic approach in ethical analysis would challenge ethicists to pay greater attention to how moral concepts are embedded in social practice and how biomedical practitioners and institutional patterns shape the production and experience of ethical dilemmas. ${ }^{36}$

Value neutrality is untenable in a "real-world" bioethics. But anthropologists can effect change both directly (in communication with patients and practitioners) and by advocating for structural change that might have broader impacts: for example, decentralization of primary care services might allow patients to develop therapeutic relationships and alliances that would limit disruptive behavior; intake and consent-gathering procedures that incorporate a larger network of those invested might forestall later crises and disputes.

What we need are theories of bioethics that do not reproduce, in an unexamined way, the assumptions of a single cultural paradigm; we would benefit from forms of pedagogy that integrate anthropological and other social science perspectives-and the incorporation of ethnographic techniques in ethical practice-to situate problems in biomedical, familial, interpersonal contexts. There are no simple and formulaic models that can address diverse cultural differences. We are suggesting the need for further cross-disciplinary talk about "how to talk," with the ultimate objective of generating informed and collaborative negotiation of critical life issues.

\section{Notes}

1. Kleinman AM. Patients and Healers in the Context of Culture: An Exploration of the Borderland between Anthropology, Medicine, and Psychiatry. Berkeley: University of California Press; 1980; Kleinman AM, Eisenberg L, Good BJ. Culture, illness, and care: Clinical lessons from anthropologic and cross-cultural research. Annals of Internal Medicine 1978;99:25-58.

2. Beauchamp T, Childress J. Principles of Biomedical Ethics, 5th ed. New York: Oxford University Press; 2001.

3. See note 1, Kleinman 1980; Helman CG. Culture, Health and Illness, 4th ed. New York: Arnold Press; 2000.

4. Huprich SK, Fuller KM, Schneider R. Divergent ethical perspectives on the duty-to-warn principle with HIV patients. Ethics \& Behavior 2003;13:263-78; Curlin F, Burck R. Patient counseling and matters of conscience. Virtual Mentor [Internet]. 2005. Available from: http:// www.ama.assn.org/ama/pub/category/14980; McKneally MF. The role of ethical principles in health care and the implications for ethical codes. Journal of Medical Ethics 1999;25:394-8. Review 5, of Alexander Limentani's essay on Beauchamp and Childress; Schmidt-Felzmann $\mathrm{H}$. Pragmatic principles-Methodological pragmatism in the principle-based approach to bioethics. Journal of Medical Philosophy 2003;28(5-6):581-96; O'Neill O. Practical principles, practical judgment. Hastings Center Report 2001;31(4):15-23; Tanenbaum S. Say the right thing: communication and physician accountability in the era of medical outcomes. In: Boyle PJ, ed. Getting Doctors to Listen: Ethics and Outcomes Data in Context. Hastings Center Studies in Ethics. Washington, D.C.: Georgetown University Press; 2000:204-25.

5. See note 4, O'Neill 2001:15.

6. See note 4, O'Neill 2001:15. 


\section{Carolyn Sargent and Carolyn Smith-Morris}

7. Annas GJ. "Culture of life" politics at the bedside-The case of Terri Schiavo. New England Journal of Medicine 2005;352(16):1710-5.

8. See note 7, Arnas 2005:1715.

9. See note 2, Beauchamp and Childress 2001:2.

10. See note 2, Beauchamp and Childress 2001:12.

11. See note 2, Beauchamp and Childress 2001:3.

12. See note 2, Beauchamp and Childress 2001:404.

13. see note 2, Beauchamp and Childress 2001:13.

14. Marshall P, Koenig B. Accounting for culture in a globalized bioethics. Journal of Law, Medicine \& Ethics 2004;32:252-66 at p. 253.

15. See note 14, Marshall and Koenig 2004:253.

16. Korbin's 1984 volume, Child Abuse and Neglect in Cross-Cultural Perspective, is an early classic that raised questions concerning global variability in what is perceived to constitute abuse and neglect (Korbin J. Child Abuse and Neglect: Cross-Cultural Perspectives. Berkeley: University of California Press; 1984). The on-going debate regarding female genital cutting continues this theme; some scholars argue that FGC represents child abuse, the mutilation of a child's body without her consent, while others contend that as a normative cultural practice, it should not be categorized as "abusive" (Shell-Duncan B, Hernlund Y, eds. Female "Circumcision" in Africa. Boulder and London: Lynn Reiner Publishers; 2000). Herdt's analysis of masculinity rituals among the Sambia of Highland New Guinea describes the local rationale for ritualized oral sex between older men and young boys as part of male initiations (Herdt G. Rituals of Manhood: Male Initiation in Papua New Guinea. Berkeley: University of California Press; 1982). This practice is considered requisite for boys to become men, although a Euro-American assessment might classify the tradition as coercive or a form of pedophilia. Also in New Guinea, ethnographers have documented "gang rape" of women who have breached a taboo and seen the interior of the "men's house" or have witnessed rituals at which women's presence is prohibited. This "punishment" of women is legitimized as a response to seriously inappropriate behavior. The concept of rape does exist, but this particular scenario, culminating in forced sex between a group of men and an unwilling woman, would not be defined as such.

17. See note 3, Helman 2000:103.

18. See note 14, Marshall and Koenig 2004:255.

19. See note 2, Beauchamp and Childress 2001:61-2.

20. See note 2, Beauchamp and Childress 2001:63.

21. See note 2, Beauchamp and Childress 2001:4.

22. Barnard A. History and Theory in Anthropology. Cambridge: Cambridge University Press; 2000:99.

23. Marshall P. Anthropology and bioethics. Medical Anthropology Quarterly 1992;6(1):49-73 at p. 50.

24. Koenig B. Cultural diversity in decision-making about care at the end of life. In: Field MJ, Cassel, CK, eds. Approaching Death: Improving Care at the End of Life. Washington, D.C.: Institute of Medicine; 1997:363-82.

25. Smith-Morris C. False expectations? Expectations vs. probabilities for dying. Omega Journal of Death \& Dying 2000;41(3):157-85.

26. Kaufman S. Clinical narratives and ethical dilemmas in geriatrics. In: Hoffmaster B, ed. Bioethics in Social Context. Philadelphia: Temple University Press; 2001:12-39 at p. 17.

27. Jordan B. Authoritative knowledge and its construction. In: Davis-Floyd R, Sargent CF, eds. Childbirth and Authoritative Knowledge: Cross-Cultural Perspectives. Berkeley: University of California Press; 1997:55-80; Davis-Floyd R, Sargent CF. Childbirth and Authoritative Knowledge: Cross-Cultural Perspectives. Berkeley: University of California Press; 1997.

28. See note 3, Helman 2000:86.

29. See note 3 , Helman 2000:96.

30. Hunter KM. Doctors' Stories: The Narrative Structure of Medical Knowledge. Princeton, N.J.: Princeton University Press; 1991.

31. Hoffmaster B. Bioethics in Social Context. Philadelphia: Temple University Press; 2001.

32. See note 3, Helman 2000:86.

33. See note 1, Kleinman 1980:58.

34. See note 23, Marshall 1992:49.

35. For an insightful discussion of this history see Stevens MLT. Bioethics in America: Origins and Cultural Politics. Baltimore: The Johns Hopkins University Press; 2000.

36. Jennings B. Ethics and ethnography in neonatal intensive care. In: Weisz G, ed. Social Science Perspectives on Medical Ethics. Philadelphia: University of Pennsylvania Press; 1990:261-73 at p. 269; also see note 23, Marshall 1992:53. 